\title{
Coronavirus-induced severe acute respiratory syndrome (sars) as
} a possibile expression of fatty acid amide hydrolase (faah) hyperactivation and possible therapeutic role of faah inhibitors in covid19-induced sars.

\author{
Paolo Lissoni ${ }^{1 *}$, Franco Rovelli ${ }^{1}$, Francesco Pelizzoni ${ }^{2}$, Arianna Lissoni ${ }^{1}$, Giuseppe Di Fede ${ }^{1}$ \\ ${ }^{1}$ Institute of Biological Medicine, Milan, Italy. \\ ${ }^{2}$ Niguarda Hospital, Milan, Italy \\ *Corresponding author: Paolo Lissoni, Institute of Biological Medicine, Milan, Italy. \\ Received date: July 12, 2020; Accepted date: July 28, 2020; published date: August 11, 2020
}

Citation: Paolo Lissoni, Franco Rovelli, Francesco Pelizzoni, Arianna Lissoni, Giuseppe Di Fede. Coronavirus-induced severe acute respiratory syndrome (sars) as a possibile expression of fatty acid amide hydrolase (faah) hyper-activation and possible therapeutic role of faah inhibitors in covid19-induced sars.. J Clinical Research and Reports, 5(2); DOI:10.31579/2690-1919/108

Copyright: () 2020 Paolo Lissoni. This is an open access article distributed under the Creative Commons Attribution License, which permits unrestricted use, distribution, and reproduction in any medium, provided the original work is properly cited.

\section{Abstract}

It has appeared that the acute respiratory distress induced by COVID 19 is mainly depending on the excessive host inflammatory response, consisting of an inappropriate secretion of several inflammatory cytokines, namely IL-17A, IL-6 and TNF-alpha, rather than to a direct viral-induced tissue damage. Moreover, it is known that the inflammatory response is physiologically under a neuroendocrine regulation, namely played by the endogenous cannabinoid system. COVID 19-related cannabinoid reduced activity would be due to a virus-induced activation of the enzyme responsible for cannabinoid degradation, the fatty acid amide hydrolase (FAAH). Then, FAAH inhibitors could exert a therapeutic role in COVID 19 infection.

Key Words: Cannabinoid system; COVID 19; Fatty acid amide hydrolase (FAAH); FAAH inhibitors

\section{Introduction}

The endocannabinoid system (ECS) represents an endogenous defense complex to protect against an exaggerated inflammatory biological reaction in both tumor growth and cardiovascular disorders (1). The ECS may produced both $\mathrm{CB} 1$ and $\mathrm{CB} 2$ agonists, and non-cannabinoid agents $(2,3)$. The main endogenous cannabinoid agonists are the arachidonylethanol-amide (AEA) and the 2-arachidonyl-glicerol (2-AG) (1-3), while the main non-cannabinoid agonists are the palmitoyl-ethanol-amide (PEA), and the oleoyl-ethanol-amide (OEA) (4), which may be considered as the endogenous equivalents of the non-cannabinoid agonist and the non-psychoactive agent of Cannabis, the cannabidiol (CBD) (13 ). The main enzyme involved in the metabolic destruction of cannabinoids is the fatty acid amide hydrolase (FAAH), as well as monoacylglycerol lipase (MAGL) (1-3). Moreover, it has been shown that both CBD and PEA, even though they are not cannabinoid agonists, may allow an increase in the endogenous content of cannabinoid agonists by acting as FAAH inhibitors. Finally, it has been demonstrated that a hyperactivation of FAAH activity may allow to an enhanced inflammatory response, as well to an increased predisposition to cancer onset and development (5). FAAH cellular concentrations would increase with age, then this finding could explain the tendency to an exaggerated inflammatory response against several causes, including the viral infections (1-3). The higher immunobiological response in aged subjects would be also to age-related increase in TH17 lymphocyte activity (6), with a consequent enhanced inflammatory reactivity, in association with a decline in that of regulatory $\mathrm{T}$ lymphocytes ( $\mathrm{T}$ reg) (7), which in contrast counteract the inflammatory response. The inhibitory action of FAAH inhibitors on the intensity of the inflammatory response may be amplified by the pineal hormone melatonin (MLT) (8), by suggesting the existence of a functional axis between brain cannabinoid system and pineal gland (9).

\section{Coronavirus-Endocannabinoid System Interactions}

It is known that the ECS plays a depend on a fundamental role in the perception of pleasure, including that related to the taste (1-3). Moreover, it is a common evidence that one the main early COVID 19-related symptoms is the loss of taste. Then, the loss of taste induced by the infection of COVID 19, also called SARS-COV-2, could depend on an acute ECS deficiency. Finally, it is known that the spike glycoprotein of COVID 19, which is located on the outer envelope of the virion and constituted by two subunits, binds to host receptor angiotensin-converting enzyme-2 (ACE-2) through its receptor binding domain (RBD)(10). Then, Coronavirus infection would allow an exaggerated immuneinflammatory response (11). Since FAAH hyper-activation may also allow an excessive immune-inflammatory responses, coronavirus infection might induce an excessive inflammatory response by determining an ECS deficiency. In other words, viral spike glycoproteinACE-2 interactions would allow a hyper-stimulation of FAAH activity, 
with a consequent failure in ECS function, which has been proven to predispose to cardiopulmonary complications (12). In more detail (5), FAAH hyper-activation would allow a down-regulation of tissue inhibitor of matrix metalloproteinase-1 (MP-1), with a consequent enhanced MP1 -induced alterations of the intercellular matrix and a stimulation of the angiogenetic processes (5).

\section{Possible Block of Covid 19-Induced Inflammatory Response By A Neuroimmune Approach}

If the main problem of COVID 19 infections is the control of host excessive inflammatory response, namely due to TNF-alpha, IL-6 and IL17A hyper-production, because of the fundamental role of FAAH activity in the generation of the inflammatory response, one simple way to reduce host exaggerated inflammatory response could consist of the inhibition of FAAH activity, which may be achieved by the same endogenous FAAH inhibitors, such as PEA (4), by CBD (1-3), or by the most recent FAAH inhibitors, including arachidonoylglycerol (AA-5HT), URB597 (5), and SA-57, which may inhibit both FAAH and MAGL (13). Mu-opioid agonists would also inhibit FAAH (13). Doses and schedule of administration will be established by controlled clinical studies.

\section{Conclusions}

COVID-19 infection may be interpreted as an in vivo dramatic examination of the immunological knowledgements of the human Sciences, since the terrible lethality induced by its infection is the consequence of a profound alteration of the interactions between host immune-inflammatory response and its neuroendocrine regulation. The lacked evolution of the Immunology into the Psycho-neuro-immunology is the main cause of the inappropriate therapeutic response to COVID 19 infection and the consequent acute inflammatory respiratory failure.

\section{References}

1. Russo EB. Clinical endcannabinoid deficiency (CECD). Neuroendocrinol Lett 25: 31-39, 2004.

2. Grotenhermen F. Pharmacology of cannabinoids. Neuroendocrinol Lett 25:14-23, 2004.

3. Nagarkatti P, Pandey R,Rieder SA, Hedge VL,Nagarkatti M. Cannabinoids as novel anti-inflammatory drugs. Furure Med Chem 1: 1333-1349, 2009.
4. Di Marzo V, Melck D, Orlando P, Bisogno T, Zagoory O, Bifulco M, Vogel Z, De Petronillis L. Palmitoyl-ethanol-amide inhibits the expression of fatty acid amide hydrolase and enhances the anti-proliferative effect of anandamide in human breast cancer cells. Biochem J 358: 249-255, 2001.

5. Winkler K, Ramer R, Dithmer S, Ivanov I, Merkors J, Hinz B. Fatty acid amide hydrolase inhibitors confer anti-invasive and antimetastatic effects on lung cancer cells. Oncotarget 7: 15047-15064, 2016.

6. Tesar BM, Du W, Shirali AC, Walker WE, Shen H, Goldstein DR. Aging augments IL-17 T cell alloimmune responses. Am J Transplant 9: 54-63, 2009.

7. Hwang KA, Kim HR, Kang I. Aging and human CD4+ regulatory T cells. Mech Ageing Dev 130: 509-517, 2009.

8. Spadoni G, Bedini A, Furiassi L, Mari M, Mor M, Scalvini L, Lodola A, et.al. Identification of bivalent ligands with melatonin receptor agonist and fatty acid amide hydrolase (FAAH) inhibitory activity that exhibit ocular hypotensive effect in the rabbit. J Med Chem.

9. Lissoni P,Messina G, Porro G, Trampetti R, Lissoni A, Rovelli F, Cenaj V, Porta E, Di Fede G. The modulation of the endocannabinoid system in the treatment of cancer and other systemic human diseases. Glob Drugs Therap 3: 1-4, 2018.

10. Liu Z, Xiao X, Wei X, Li J, Yang J, Tan H, Zhu J, Zang Q, Wu $\mathrm{J}$, Liu L. Composition and divergence of coronavirus spike proteins and host ACE-2 receptors predict potential intermediate hosts of SARS-COV-2. J Med .

11. Li G, Fan Y, Lai Y, Han T,Li Z, Zhou P, Pan P, Wang W, Hu $\mathrm{D}$, Liu X, Zhang Q, Wu J. Coronavirus infections and immune responses. J Med Virol.

12. Cunha P, Romao AM, Mascarenhas-Melo F, Teixeira HM, Reis F. Endocannabinoid system in cardiovascular diseases: new pharmacotherapeutic opportunities. J Pharm Bioallied Sci 3: 350-360, 2011.

13. Willkerson JL, Grosh S, Mustafa M, Abdullah RA, Niphakis MJ, Cabrera R, Maldonado RL, Cravatt BF, Lichtman AH. The endocannabinoid hydrolysis inhibitor SA-57: intrinsic antinociceptive effects, augmented morphine-induced antinociception, and attenuated heroin seeking heroin in ice. Neuropharmacology 114: 156-167, 2017. 